\title{
Insomnia is associated to depressive symptoms in patients with chronic heart failure
}

\author{
Peter Johansson ${ }^{1,2}$, Anders Broström ${ }^{3,4}$ \\ ${ }^{1}$ Department of Cardiology, Linköping University Hospital, Linköping, Sweden \\ ${ }^{2}$ Department of Medicine and Care, Division of Cardiovascular Medicine, Faculty of Health Sciences, Linköping University, Linköping, \\ Sweden \\ ${ }^{3}$ Division of Clinical Neurophysiology, Linköping University Hospital, Linköping, Sweden \\ ${ }^{4}$ Department of Neuroscience and Locomotion, Faculty of Health Sciences, Linköping University, Linköping, Sweden \\ Email: anders.brostrom@hhj.hj.se
}

Received 6 January 2013; revised 26 February 2013; accepted 1 March 2013

\begin{abstract}
Introduction: Insomnia and depressive symptoms are common among patients with chronic heart failure (HF). Aim: The aim was to describe the prevalence of insomnia and depressive symptoms, as well as to examine the association between insomnia and depressive symptoms in patients with HF. Method: A crosssectional descriptive study including 212 patients with HF. All patients responded to questionnaires regarding sleeping difficulties (Uppsala Sleep InventoryChronic Heart Failure), daytime sleepiness (Epworth Sleepiness Scale) and depressive symptoms (The Mental Health Scale). Results: Patients with depressive symptoms (34\%) had, compared to those without, significantly more major complaints of insomnia as indicated by the sub-types; non-restorative sleep (66\% vs. $32 \%, \mathbf{p}<0.0001$ ), difficulty in maintaining sleep $(35 \%$ vs. $16 \%, p=0.003)$, difficulty in initiating sleep $(29 \%$ vs. $14 \%, p=0.009)$ and early morning awakenings $(25 \%$ vs. $10 \%, p=0.004)$. The odds ratio (OR) to suffer from depressive symptoms; were for; nonrestorative sleep 5.2 (CI 95\%, 2.2 - 12.3), difficulties maintaining sleep 2.5 (CI 95\%, 1.2 - 4.9), difficulties in initiating sleep 2.2 (CI 95\%, $1.1-4.4)$ and early morning awakenings 2.4 (CI 95\%, 1.1 - 5.4). When categorising insomnia into three severity groups, 1) non insomnia, 2) mild insomnia, and 3) severe insomnia, the OR for depressive symptoms for the mild insomnia and severe insomnia group were 2.2 (CI 95\%, 1.1 - 4.2) and 7.4 (CI 95\%, 2.4 - 22.8) respectively, compared to the non insomnia group. Conclusion: Insomnia is independently associated to depressive symptoms. Assessment of depressive symptoms and insomnia in patients with HF is important since treatment could be targeted to depressive symptoms only and/or to the sleep disturbance.
\end{abstract}

Keywords: Congestive Heart Failure; Insomnia
Disorders; Depressive Symptoms

\section{INTRODUCTION}

Disturbed sleep measured objectively, as well as subjectively, has been recognized as a common problem among patients with HF [1-3]. One study found that $36 \%$ of 223 patients with HF reported not getting enough sleep and $21 \%$ reported excessive daytime sleepiness [2]. Both the quality, as well as the continuity of sleep are important since these independently are associated to functional performance and to the physical and mental quality of life [4]. Reasons for this may be related to insomnia [3]. Insomnia is according to the Diagnostic and Statistical Manual of Mental Disorders described as a complaint of difficulty initiating sleep, difficulty maintaining sleep and/or a non-restorative sleep [5]. Primary insomnia means that the disturbance is due to unknown causes while secondary insomnia suggests medical, psychiatric or abuse being the causes for the disturbance [5]. Many studies also include early morning awakenings in the definition of insomnia [6]. As much as $20 \%-50 \%$ of patients with HF may suffer from insomnia [1,2,7], and complaints of difficulties maintaining sleep or insufficient sleep have been found to be the two most commonly reported disturbances [1,2]. Compared to noninsomniac HF patients, as well as to a normal population, HF patients complaining of insomnia also rate a poorer quality of life [2].

In HF secondary insomnia can be caused by physical aspect such as dyspnoea, palpitations or nocturia [8]. Pain or sleep disordered breathing can be other causes [9], however, the relationship between insomnia and sleep disordered breathing is unclear, since some studies report an association [10] whereas others not $[11,12]$. Depression can be a psychiatric cause for insomnia [13]. The diagnosis of depression (Table 1) is made upon the presence of at least one of the two core symptoms, i.e. 
Table 1. Core and secondary symptoms of depression according to American psychiatric association [5].

\begin{tabular}{c} 
Core symptoms \\
$\begin{array}{c}\text { Depressed, sad mood most of the day. } \\
\text { Markedly diminished interest or pleasure in } \\
\text { all or almost all activities most of the day, } \\
\text { nearly every day. }\end{array}$ \\
\hline Secondary symptoms \\
$\begin{array}{c}\text { Weight loss or weight gain, or decrease } \\
\text { or increase in appetite. } \\
\text { Insomnia or hypersomnia. } \\
\text { Psychomotor agitation/retardation. } \\
\text { Fatigue or loss of energy. } \\
\text { Feelings of worthlessness or } \\
\text { inappropriate guilt. } \\
\text { Diminished ability to think or concentrate } \\
\text { or ambivalence. }\end{array}$ \\
Thoughts of death or suicidal ideation. \\
\hline
\end{tabular}

sadness and loss of pleasure (anhedonic symptoms), together with at least four or more secondary symptoms [5]. Studies in aged non HF populations have shown mood disturbances to be one of the most important factors associated with sleep disturbances [14]. The link between sleep disturbances and depression is however complicated. Sleep disturbances can in some cases be a precursor to depression and in other cases, a consequence of depression [13]. Although data indicate that approximately $40 \%$ of the HF population might suffer from depression or depressive symptoms [15], only one study to our knowledge have investigated the association between insomnia and depression in patients with HF. Redeker et al. [16] reported that patients with difficulties initiating sleep and/or difficulties maintaining sleep were five time more likely to be depressed. A deeper knowledge of the associations between insomnia and depressive symptoms is needed to design patient centred interventions to improve sleep and psychological wellbeing in patients with HF. The aim of this study was therefore to describe the prevalence of insomnia and depressive symptoms, as well as to examine the association between insomnia and depressive symptoms in patients with HF.

\section{METHODS}

\subsection{Design and Setting}

This study used a cross-sectional descriptive design. Patients were recruited from one medical ward (in a university hospital), two heart failure clinics (one university and one county hospital) and five primary health care centres in the southeast of Sweden.

\subsection{Study Population}

The template is used to format your paper and style the text. All margins, column widths, line spaces, and text fonts are prescribed; please do not alter them. You may note peculiarities. For example, the head margin in this template measures proportionately more than is customary. This measurement and others are deliberate, using specifications that anticipate your paper as one part of the entire journals, and not as an independent document. Please do not revise any of the current designations.

\subsection{Uppsala Sleep Inventory-Chronic Heart Failure}

Insomnia was measured with the well-validated Uppsala Sleep Inventory-Chronic Heart Failure (USI-CHF) [2, 17]. The USI-CHF consists of 26 items of which four focuses on insomnia (i.e., difficulty initiating sleep, difficulty maintaining sleep, non-restorative sleep and early morning awakenings). The items about insomnia are to be answered on a five-point likert-type scale; from no problems (1), small problems (2), some problems (3), great problems (4), to very great problems (5). In the statistical processing and analysis of the insomnia variables of the USI-CHF scores of 1 - 3 were considered to represent "no complaints" and scores 4 and 5 "major complaints".

\subsection{Epworth Sleepiness Scale}

Excessive daytime sleepiness was measured with the well-validated Epworth Sleepiness Scale (ESS) $[18,19]$. The 8 items focuses the expectation of "dozing" in a variety of situations. The probability ratings are zero (0), slight (1), moderate (2), or high (3) in the hypothetical situations. The ratings can be summarized to a total score of 24 , with a cut-off value of $>10$ indicating excessive daytime sleepiness.

\subsection{Mental Health Scale}

In the present study depressive symptoms was measured by the Mental Health Scale (MH-5), from the 36-Item Short Form Health Survey [20]. The 5 items focuses the anhedonic aspect of depression (i.e., sadness and loss of pleasure) and the scores can range from 0 (the worst) to 100 (best mental health). With a cut-off point of $\leq 60$ the MH-5 seems valid and reliable to use in the screening for depressive symptoms indicating a high probability of a diagnosis of major depression [21-23]. The MH-5 can be said to assess a depressive symptomatology which might indicate the presence of different types of depressive disorders such as subsyndromal or minor depression, dysthymia or major depression [15]. Due to this the term 
depressive symptoms is used in this study. Hence, participants with a score $\leq 60$ on the MH-5 scale were therefore labeled as having depressive symptoms and those with a score $>60$ were labeled as having no depressive symptoms.

\subsection{Data Collection}

HF nurses and primary health care nurses acted as coordinators at each center. Eligible patients with HF were found through a screening process on wards and heart failure clinics at the two hospitals and through the Diagnosed Related Groups register at each of the five primary health care centers. The medical records were scrutinized by an experienced heart failure nurse, together with one of the nurse-coordinators and a physician. All participants gave their informed written consent. The study protocol was approved by the ethics committee at the faculty of health sciences, University of Linköping, Sweden, and is in accordance with the provisions of the Helsinki declaration.

\subsection{Statistical Processing and Analysis}

Descriptive statistics were used to describe differences between the HF population with and without depressive symptoms. Categorical variables were analyzed with the Chi-square test. Continuous variables, if normally distributed, were analyzed with the Student's t-test (2-tailed) and presented as means and standard deviation (SD). The Mann-Whitney non-parametric test were used on skewed continuous variables, and presented as the median (md) and the 25 th 75 th percentiles. To determine the interrelationship between difficulties initiating sleep, difficulties maintaining sleep, early morning awakenings and non restorative sleep a Spearman's rank correlation analysis was done. Binary regression modeling was used to analyze the independent relation between depressive symptoms and the insomnia variables. The model was adjusted for covariates that in Table 2 had a univariate $p$ value $<0.15$ (i.e. NYHA class). The significance level was set at 0.05 . Statistical computing was done with the SPSS 18.0.

Table 2. Characteristics of HF patients $(n=212)$ and unvariate differences between patients with and without DS.

\begin{tabular}{|c|c|c|c|c|}
\hline & Total group & No DS $n=140$ & $\mathrm{DS} n=72$ & $\mathrm{p}$ \\
\hline Sex, male, n (\%) & $129(61)$ & $87(41)$ & $42(20)$ & 0.59 \\
\hline Age mean, (SD) & $76 \pm 9$ & $76 \pm 8$ & $75 \pm 11$ & 0.40 \\
\hline Living with a partner, $\mathrm{n}(\%)$ & $89(42)$ & $55(39)$ & $34(38)$ & 0.27 \\
\hline MH-5 score, md, (Q25 - Q75) & $76(56-88)$ & $84(76-92)$ & $52(40-56)$ & 0.001 \\
\hline NYHA-class & & & & 0.006 \\
\hline II, n (\%) & $86(40)$ & $65(46)$ & $21(29)$ & \\
\hline III, n (\%) & $114(54)$ & $70(50)$ & $44(61)$ & \\
\hline $\mathrm{IV}, \mathrm{n}(\%)$ & $12(6)$ & $5(4)$ & $7(10)$ & \\
\hline \multicolumn{5}{|l|}{ Aetiology } \\
\hline IHD, n (\%) & $94(44)$ & $61(44)$ & $33(46)$ & 0.75 \\
\hline MI, n (\%) & $87(41)$ & $56(40)$ & $31(43)$ & 0.67 \\
\hline HT, n (\%) & $73(34)$ & $50(36)$ & $23(32)$ & 0.58 \\
\hline Diabetes, n (\%) & $53(25)$ & $36(26)$ & $17(24)$ & 0.74 \\
\hline Other, n (\%) & $29(14)$ & $19(14)$ & $10(14)$ & 0.95 \\
\hline \multicolumn{5}{|l|}{ Blood pressure } \\
\hline SBP, mean, SD & $131 \pm 22$ & $133 \pm 22$ & $127 \pm 21$ & 0.04 \\
\hline DBP, mean, SD & $71 \pm 13$ & $72 \pm 12$ & $70 \pm 13$ & 0.31 \\
\hline \multicolumn{5}{|l|}{ Sleep variables } \\
\hline DIS, n (\%) & $41(19)$ & $20(14)$ & $21(29)$ & 0.009 \\
\hline DMS, n (\%) & $48(23)$ & $23(16)$ & $25(35)$ & 0.003 \\
\hline EMA, n (\%) & $32(15)$ & $14(10)$ & $18(25)$ & 0.004 \\
\hline NRS, n (\%) & $93(44)$ & $45(32)$ & $48(66)$ & 0.0001 \\
\hline EDS, n (\%) & $46(22)$ & $27(19)$ & $19(26)$ & 0.23 \\
\hline
\end{tabular}

IHD_Ischaemic Heart Disease; MI—Myocardial Infarction; HT-Hypertension; SBP—Systolic Blood Pressure; DPB-Diastolic Blood Pressure; DISDifficulty Initiating Sleep; DMS—Difficulty Maintaining Sleep; EMA—Early Morning Awakenings; NRS—Non Restorative Sleep; EDS—Excessive Daytime Sleepiness. 


\section{RESULTS}

\subsection{Participants}

The mean age of the patients was 76 years, $61 \%$ males and $42 \%$ lived with a partner (Table 2). A total of $94 \%$ were in NYHA classes II-III. Ischaemic heart disease (44\%) mostly explained by myocardial infarction (41\%) and hypertension (34\%) were the most common causes of HF (Table 2). A total of $82 \%$ of the patients had their diagnosis of HF based on radiographic evidence and/or echocardiography.

\subsection{Prevalence of Depressive Symptoms}

Of the patients $34 \%(n=72)$ suffered from depressive symptoms. Regarding age, partnership, sex and aetiology of $\mathrm{HF}$, there were no significant differences between those with and without depressive symptoms (Table 2). A higher proportion of the non-depressed patients were in NYHA class II and a higher proportion of those with depressive symptoms were in NYHA class III $(\mathrm{p}=$ 0.006). Those with depressive symptoms had a lower systolic blood pressure $(\mathrm{p}=0.04)$ compared to those without depressive symptoms (Table 2).

\subsection{Insomnia, Excessive Daytime Sleepiness and Depressive Symptoms}

The most common insomnia type was non restorative sleep, which was reported as a major complaint by $44 \%$ of the patients. Major complaints of difficulties maintaining sleep, difficulties initiating sleep and early morning awakenings were reported by $23 \%, 19 \%$ and $15 \%$ of the patients, respectively. Patients with depressive symptoms had significantly more major complaints of non restorative sleep $(\mathrm{p}<0.0001)$, difficulties maintaining sleep $(p=0.003)$, difficulties initiating sleep $(p=0.009)$ and early morning awakenings $(p=0.004)$ than to those without depressive symptoms (Table 2). Fully one fifth $(22 \%)$ reported excessive daytime sleepiness. There was no significant difference in excessive daytime sleepiness reported between the patients with and without depressive symptoms (Table 2).

\subsection{Association between Insomnia and Depressive Symptoms}

To examine which of the insomnia variables that independently was associated to depressive symptoms, all variables were included in the same regression model. However in this analysis none of the insomnia variables became significant. This can probably be explained because of an interrelationship between the insomnia variables (Table 3). Therefore separate models including the insomnia variables were calculated with adjustments for
NYHA classes, with NYHA class II as the referent. In the unadjusted model all insomnia variables had a significant relation to depressive symptoms. Major complaints of non restorative sleep more than five folded the OR to have depressive symptoms. The OR to have depressive symptoms for major complaints of early morning awakenings almost increased by three fold, while the OR for major complaints of difficulties initiating sleep and difficulties maintaining sleep increased more than two fold respectively (Table 4). After adjustment for NYHA class, all insomnia variables were still significantly related to depressive symptoms. In the adjusted model major complaints of non restorative sleep more than five folded the odds to suffer from depressive symptoms. Reports of major complaints of early morning awakenings, difficulties initiating sleep and difficulties maintaining sleep respectively more than doubled the odds for having depressive symptoms. However, NYHA class by itself had a significant association to depressive symptoms only in the models for difficulties initiating sleep and difficulties maintaining sleep (Table 4).

Since many patients suffer from more than one type of insomnia, we studied the relationship between the amount of insomnia subtypes and depressive symptoms (i.e., the higher degree of insomnia the higher proportion of patients with depressive symptoms). In this model insomnia was categorized into three groups; the first group included those who did not report to suffer from a major complaint in any of the insomnia groups (non insomnia), the second group (mild insomnia) included those that had a major complaint in one or two of the insomnia groups; and the third group (severe insomnia) consisted of those who had a major complaint in three or four of the insomnia groups. The percentages of patients with depressive symptoms increased proportionally from $24 \%$ in the no insomnia group to $74 \%$ in the severe insomnia group (Figure 1). Compared to the non insomnia group, the patients with mild and severe insomnia had more than two fold and seven fold increased risk respectively to suffer from depressive symptoms. In the adjusted model,

Table 3. Interrelationship between the insomnia subtypes in patients with HF.

\begin{tabular}{ccccc}
\hline & DIS & DMS & EMA & NRS \\
\hline DIS & - & $0.4^{* *}$ & $0.260^{* *}$ & $0.349^{* *}$ \\
DMS & $0.449^{* *}$ & - & $0.338^{* *}$ & $0.395^{* *}$ \\
EMA & $0.260^{* *}$ & $0.338^{* *}$ & - & $0.320^{* *}$ \\
NRS & $0.349^{* *}$ & $0.395^{* *}$ & $0.320^{* *}$ & - \\
\hline
\end{tabular}

DIS - Difficulty Initiating Sleep; DMS - Difficulty Maintaining Sleep; EMAEarly Morning Awakenings; NRS - Non Restorative Sleep; EDS-Excessive Daytime Sleepiness. ${ }^{* *} \mathrm{p}<0.01$. 
Table 4. Odds ratios (OR) for the association between the insomnia variables and depressive symptoms (DS) in patients with HF.

\begin{tabular}{|c|c|c|c|c|}
\hline & $\begin{array}{c}\text { Unadjusted } \\
\text { OR (CI 95\%) }\end{array}$ & $\mathrm{p}$ & $\begin{array}{c}\text { Adjusted } \\
\text { OR (CI 95\%) }\end{array}$ & $\mathrm{p}$ \\
\hline DIS & $2.5(1.2-4.9)$ & 0.011 & $2.2(1.1-4.4)$ & 0.03 \\
\hline \multicolumn{5}{|l|}{ NYHA } \\
\hline II & & & ref & \\
\hline III & & & $1.7(0.9-3.2)$ & 0.09 \\
\hline IV & & & $3.9(1.1-13.9)$ & 0.034 \\
\hline DMS & $2.7(1.4-5.2)$ & 0.003 & $2.5(1.2-4.9)$ & 0.007 \\
\hline \multicolumn{5}{|l|}{ NYHA } \\
\hline II & & & ref & \\
\hline III & & & $1.7(0.9-3.3)$ & 0.08 \\
\hline IV & & & $4.1(1.1-14.7)$ & 0.02 \\
\hline EMA & $2.9(1.3-6.4)$ & 0.005 & $2.4(1.1-5.3)$ & 0.03 \\
\hline \multicolumn{5}{|l|}{ NYHA } \\
\hline II & & & ref & \\
\hline III & & & $1.8(0.9-3.3)$ & 0.07 \\
\hline IV & & & $3.05(0.2-11.2)$ & 0.09 \\
\hline NRS & $5.9(2.5-13.9)$ & 0.001 & $5.2(2.2-12.3)$ & 0.001 \\
\hline \multicolumn{5}{|l|}{ NYHA } \\
\hline II & & & ref & \\
\hline III & & & $1.67(0.8-3.1)$ & 0.12 \\
\hline IV & & & $3.12(0.8-11.7)$ & 0.09 \\
\hline
\end{tabular}

DIS - Difficulty Initating Sleep; DMS - Difficulty Maintaining Sleep; EMAEarly Morning Awakenings; NRS-Non Restorative Sleep; EDS-Excessive Daytime Sleepiness.

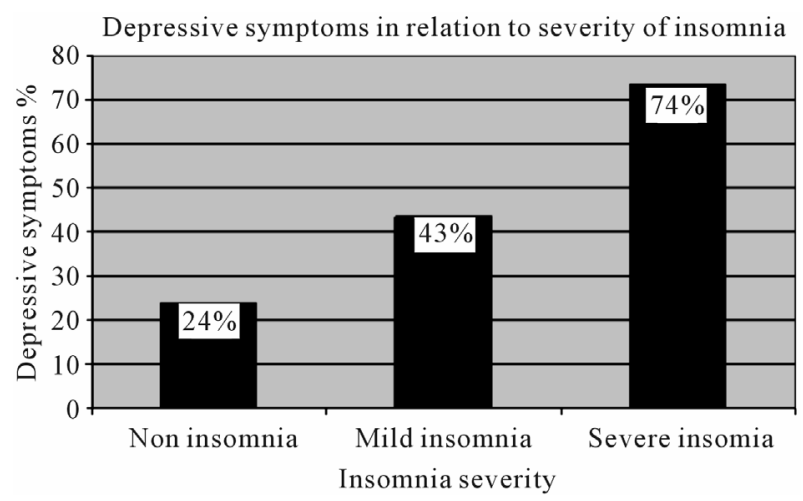

Figure 1. Proportion (\%) of patients with depressive symptoms (DS) in relation to insomnia severity. The non insomnia group includes patients not suffering of insomnia $(n=130)$. The mild insomnia $(n=62)$ group includes patients suffering from major complaint in one or two of the insomnia variables respectively. The severe insomnia group $(\mathrm{n}=19)$ includes patients suffering from major complaint in three or all of the insomnia variables.
NYHA class did not have any significant relation to depressive symptoms (Table 5).

\section{DISCUSSION}

The main findings in this study were that one third of the HF patients suffered from depressive symptoms. Insomnia was common, with non restorative sleep reported by $32 \%$ of those without and by $66 \%$ of those with depressive symptoms. The association between insomnia and occurrence of depressive symptoms was strong. The OR for HF patients with severe insomnia to suffer from depressive symptoms was more than seven folded.

\subsection{Prevalence of Depressive Symptom and Insomnia}

The prevalence of depressive symptoms in this study was $34 \%$, which is comparable with the reported median prevalence of $42 \%$ found in studies that have used dimensional depression screening tools [15]. Of the patients in the present study $44 \%$ complained of non restorative sleep, while $23 \%, 19 \%$ and $15 \%$ of the patients complained of difficulties maintaining sleep, difficulties initiating sleep and early morning awakenings, respectively. Patients with depressive symptoms also reported more insomnia. Principe-Rodriguez et al. [7] found that $31 \%$ of the HF patients presented symptoms of insomnia, but the different insomnia types were not described. Another study found $37 \%, 35 \%$ and $36 \%$ of the patients reporting difficulties initiating sleep, difficulties maintaining sleep and early morning awakenings [1]. This is higher rates than in our study. However, the $44 \%$ who

Table 5. Odds ratios (OR) for the association between severity of insomnia and depressive symptoms (DS).

\begin{tabular}{|c|c|c|c|c|}
\hline Insomnia & $\begin{array}{l}\text { Unadjusted OR } \\
\text { (CI 95\%) }\end{array}$ & $\mathrm{p}$ & $\begin{array}{l}\text { Adjusted OR } \\
\text { (CI 95\%) }\end{array}$ & $\mathrm{p}$ \\
\hline $\begin{array}{l}\text { Non insomnia, } \\
\quad(\mathrm{n}=130)\end{array}$ & ref & & ref & \\
\hline $\begin{array}{l}* * \text { Mild insomnia, } \\
\quad(\mathrm{n}=62)\end{array}$ & $2.5(1.3-4.7)$ & 0.006 & $2.2(1.1-4.2)$ & 0.01 \\
\hline $\begin{array}{l}{ }^{* * *} \text { Severe insomnia, } \\
(\mathrm{n}=19)\end{array}$ & $8.9(2.9-26.8)$ & 0.001 & $7.4(2.4-22.8)$ & 0.001 \\
\hline \multicolumn{5}{|l|}{ NYHA } \\
\hline II & & & ref & \\
\hline III & & & $1.5(0.8-2.9)$ & 0.19 \\
\hline IV & & & $2.6(0.7-10.0)$ & 0.15 \\
\hline
\end{tabular}

${ }^{*}$ The group includes patients not suffering of insomnia (i.e. DIS, DMS, EMA, NRS); ${ }^{* *}$ The group includes patients suffering from major complaint in one or two of the insomnia variables; ${ }^{* * *}$ The group includes patients suffering from major complaint of three or all of the insomnia variables. 
reported non restorative sleep in our study are at the same level as those who reported that they felt tired or fatigued in the morning at least 3 - 4 times/week in Principe-Rodriguez et al. [7], and the $45 \%$ who reported insufficient sleep in the study of Redeker and Stein [1]. In total this indicates, that about $15 \%-30 \%$ of the patients with HF suffer from insomnia and that almost $50 \%$ still are sleepy and fatigued when they wake up in the morning. The high prevalence of insomnia found also suggests that a large proportion of these patients also are at risk for suffering from depressive symptoms.

\subsection{The Association between Depressive Symptoms and Insomnia}

We found a strong association between insomnia and depressive symptoms. The OR to suffer from depressive symptoms for patients complaining of difficulties initiating sleep, difficulties maintaining sleep and early morning awakenings increased by more than two fold. These rates corresponds almost the figures found by LeesmanLeegte et al. [24] who reported the OR between sleep disturbance and depressive symptoms to be three folded. However, in the study of Redeker et al. [1] the OR between difficulties initiating sleep and/or difficulties maintaining sleep and depressive symptoms was five folded [16]. In our study a five folded increased risk was found for the association between non restorative sleep and depressive symptoms. One explanation for this may be explained by that fact that non restorative sleep might include feelings of fatigue and tiredness, which both are symptoms of HF without the co-existence of a sleep disturbance. However, NYHA class, a measure of functional capacity, had no significant relation to depressive symptoms in the separate non restorative sleep model. Moreover the relationships between insomnia and depressive symptoms persisted after that NYHA class was taken into consideration. This corresponds to the study of Redeker et al. [16] who also reported the relationship between depression and insomnia to persist after adjustments for clinical variables such as NYHA class and ejection fraction. In another study patient's perception of their sleep explained $30 \%$ of the variance in mental health compared to the $15 \%$ explained by NYHA-class, age, gender and co-morbidity [25]. Hence, insomnia in HF patients may not only be a marker for severity of HF. Furthermore, in the present study we also analysed the association between severity of insomnia and depressive symptoms. Those with severe insomnia had a seven fold increased risk to suffer from depressive symptoms. This has not before been reported in patients with HF highlights the importance of interventions such as cognitive behavioural therapy (CBT) that can be used to target both insomnia and depressive symptoms. In older adults with arthritis and insomnia, CBT improved sleep as well as pain [26]. In patients with HF, CBT has been found to improve depressive symptoms and health-related quality of life [27,28]. However, the numbers of CBT studies performed on patients with HF are few and none have examined of the effects on sleep disturbances and depressive symptoms.

In future studies it is of importance to consider that there are several different factors that can cause difficulties initiating sleep, difficulties maintain sleep and early morning awakenings (i.e. secondary insomnia). Theoretically, insomnia can be described as the combination of sleep interfering and sleep interpreting processes [29]. The sleep interfering processes include different types of arousals (i.e., physiological, emotional and cognitive). A physiological arousal may be explained by an elevated baseline level of the stress-response system, as well as a slow return to the baseline level after being aroused. Emotional and cognitive arousals may be explained by a chronic tendency to worry, an elevated response to stressful events, problems, or increased dysphoric mood. The sleep interpreting processes imply that the insomniaacs' perceptions of their own sleep are misinterpreted [29]. Sleep interfering processes leading to negative thoughts and anxiety, i.e., emotional and cognitive arousal in patients with $\mathrm{HF}$ can be can be associated with problems to perform activities in daily life, thoughts about HF including deterioration, medication and an unknown life situation $[8,16]$. Dysrhythmia, coughing and dyspnoea or have also been described causing a sense of panic during the night. Nocturia or inability to sleep in a supine position can also cause nightly awakening and be possible sleep interfering processes $[8,30]$.

However, not all patients with a disturbed sleep will develop insomnia and/or depressive symptoms. In an elderly non HF population factors describing a psychological vulnerability (i.e., somatisation, intrusive and irresistible thoughts and worries) were associated to insomnia [31]. In another study of non-HF patients showed that insomniacs and good sleepers did not differ in the frequency of experienced daily stressors. The difference was in how they coped and perceived the stressors in daily life [32]. Poor psychological functioning in elderly people with and without HF has been found to mediate the association between insomnia and health related quality of life [33]. Thus, an HF patient who complains of insomnia should therefore be carefully evaluated for presence of depressive symptoms with common and validated specific instruments for insomnia and depression $[15,34,35]$. Treatment of insomnia in clinical practice often includes sleep-medications. However, in elderly with or without HF sleep-medication should be used carefully. This is because of the risk for polypharmacy and side-effects, such as sedation, drowsiness or somno- 
lence [36]. Moreover, sleep medications have in elderly, independent of insomnia, been found to cause depression [37]. CBT (e.g., reduction of dysfunctional believes about sleep) can therefore be preferable. CBT studies in HF populations are missing but in other populations face to face CBT interventions have been successfully $[38,39]$. In a cardiovascular setting the accessibility of face to face CBT is limited due to lack of competence in CBT among the personnel. CBT provided by the internet has shown promising results to reduce insomnia as well as depressive symptoms [40-42].

\section{STUDY LIMITATIONS}

Because this study had a cross-sectional design it is not possible to determine causal relationship between insomnia and depressive symptoms. Identification of depression can be made by categorical and/or dimensional instruments. Categorical instrument can be used by a trained interviewer to decide whether the symptom profile corresponds to an inclusion or exclusion of a diagnosis of depression. In contrast, dimensional instruments are not diagnostic tools. These are based on self-reports and ranks the patients symptom profile on a continuum, and if above a certain threshold, there is a high probability for a diagnosis of depression [15]. The use of a dimensional depression screening tool (i.e., the MH-5) does not allow us to assess a clinical diagnosis of depression. However dimensional depression screening tools are widely accepted and frequently used [15,43]. Another problem with dimensional depression screening tools is that these might indicate false depressed cases due to the fact that some symptoms such as loss of appetite, fatigue and sleeplessness occur in depression as well as in HF. However, the MH-5 measures the anhedonic symptoms of depression only [20], which might reduce the problem with a false strong relationship between insomnia and depressive symptoms in the present study. The ESS is a self-assessment tool in which the individual evaluates his/her expectation of "dozing" in a variety of situations. A problem can be individual variations in relation to age, gender, habits, or other aspects that affects napping. However, validation studies using differential item functioning (i.e., analyses focusing on if a subject's response to the item is affected by other aspects than that which the test is intended to assess) have found only minor effects of age in three of the items included in the ESS. The mentioned items were, inactive in a public place (item 3), passenger in a car (item 4), and item 8 focusing on "dozing" when a car has stopped in traffic [44]. Another limitation is that at the time of inclusion data regarding existence and/or treatment of SDB, as well as if the patients were prescribed antidepressant or hypnotic medication were not collected.

\section{CONCLUSION}

This study found that insomnia as indicated by difficulties initiating sleep, difficulties maintaining sleep, early morning awakenings and non restorative sleep was independently associated to depressive symptoms in patients with HF. The study also revealed that the higher severity of insomnia, the higher was the risk of the patient to suffer from depressive symptoms. The relation between insomnia and depressive symptoms is complex and probably bi-directional. Assessment of sleep disturbances and depressive symptoms in patients with HF is therefore important, since treatment could be targeted to depressive symptoms only and/or to the sleep disturbances. Therefore more research is needed to study the relation between as well as the treatment of sleep disturbances and depressive symptoms in patients with HF.

\section{ACKNOWLEDGEMENTS}

The authors wish to thank the Health Research Council in the SouthEast of Sweden Grant no F2004-233 for financial support.

\section{REFERENCES}

[1] Redeker, N.S. and Stein, S. (2006) Characteristics of sleep in patients with stable heart failure versus a comparison group. Heart Lung, 35, 252-261. doi:10.1016/j.hrtlng.2005.10.007

[2] Brostrom, A., Stromberg, A., Dahlstrom, U. and Fridlund, B. (2004) Sleep difficulties, daytime sleepiness, and healthrelated quality of life in patients with chronic heart failure. Journal of Cardiovascular Nursing, 19, 234-242.

[3] Brostrom, A. and Johansson, P. (2005) Sleep disturbances in patients with chronic heart failure and their holistic consequences-what different care actions can be implemented? European Journal of Cardiovascular Nursing, 4, 183-197. doi:10.1016/j.ejcnurse.2005.04.005

[4] Redeker, N.S. and Hilkert, R. (2005) Sleep and quality of life in stable heart failure. Journal of Cardiac Failure, 11, 700-704. doi:10.1016/j.cardfail.2005.07.003

[5] American Psychiatric Association (1994) Diagnostic and statistical manual of mental disorders. 4th Edition, American Psychiatric Association, Washington DC.

[6] Ohayon, M.M. (2002) Epidemiology of insomnia: What we know and what we still need to learn. Sleep Medicine Reviews, 6, 97-111. doi:10.1053/smrv.2002.0186

[7] Principe-Rodriguez, K., Strohl, K.P., Hadziefendic, S. and Pina, I.L. (2005) Sleep symptoms and clinical markers of illness in patients with heart failure. Sleep Breath, 9, 127-133. doi:10.1007/s11325-005-0023-0

[8] Brostrom, A., Stromberg, A., Dahlstrom, U. and Fridlund, B. (2001) Patients with congestive heart failure and their conceptions of their sleep situation. Journal of Advanced Nursing, 34, 520-529. doi:10.1046/j.1365-2648.2001.01781.x

[9] Johansson, P., Riegel, B., Svensson, E., et al. (2012) The 
contribution of heart failure to sleep disturbances and depressive symptoms in older adults. Journal of Geriatric Psychiatry and Neurology, 25, 179-187.

[10] Skobel, E., Norra, C., Sinha, A., Breuer, C., Hanrath, P. and Stellbrink, C. (2005) Impact of sleep-related breathing disorders on health-related quality of life in patients with chronic heart failure. European Journal of Heart Failure, 7, 505-511. doi:10.1016/j.ejheart.2004.07.020

[11] Redeker, N.S., Muench, U., Zucker, M.J., et al. (2010) Sleep disordered breathing, daytime symptoms, and functional performance in stable heart failure. Sleep, 33, 551560.

[12] Johansson, P., Alehagen, U., Ulander, M., Svanborg, E., Dahlstrom, U. and Brostrom, A. (2011) Sleep disordered breathing in community dwelling elderly: Associations with cardiovascular disease, impaired systolic function, and mortality after a six-year follow-up. Sleep Medicine, 12, 748-753. doi:10.1016/j.sleep.2011.03.012

[13] Lustberg, L. and Reynolds, C.F. (2000) Depression and insomnia: Questions of cause and effect. Sleep Medicine Reviews, 4, 253-262. doi:10.1053/smrv.1999.0075

[14] Roberts, R.E., Shema, S.J. and Kaplan, G.A. (1999) Prospective data on sleep complaints and associated risk factors in an older cohort. Psychosomatic Medicine, 61, 188196.

[15] Johansson, P., Dahlstrom, U. and Brostrom, A. (2006) The measurement and prevalence of depression in patients with chronic heart failure. Progress in Cardiovascular Nursing, 21, 28-36. doi:10.1111/j.0197-3118.2006.04644.x

[16] Redeker, N.S., Jeon, S., Muench, U., Campbell, D., Walsleben, J. and Rapoport, D.M. (2010) Insomnia symptoms and daytime function in stable heart failure. Sleep, 33, 12101216.

[17] Brostrom, A., Hubbert, L., Jakobsson, P., Johansson, P., Fridlund, B. and Dahlstrom, U. (2005) Effects of longterm nocturnal oxygen treatment in patients with severe heart failure. Journal of Cardiovascular Nursing, 20, 385396.

[18] Johns, M.W. (1991) A new method for measuring daytime sleepiness: The Epworth sleepiness scale. Sleep, 14, 540-545.

[19] Johns, M.W. (1992) Reliability and factor analysis of the epworth sleepiness scale. Sleep, 15, 376-381.

[20] Ware Jr., J.E., and Sherbourne, C.D. (1992) The MOS 36-item short-form health survey (SF-36). I. Conceptual framework and item selection. Medical Care, 30, 473483. doi:10.1097/00005650-199206000-00002

[21] Friedman, B., Heisel, M. and Delavan, R. (2005) Validity of the SF-36 five-item mental health index for major depression in functionally impaired, community-dwelling elderly patients. Journal of the American Geriatrics Society, 53, 1978-1985. doi:10.1111/j.1532-5415.2005.00469.x

[22] Rumpf, H.J., Meyer, C., Hapke, U. and John, U. (2001) Screening for mental health: Validity of the MHI-5 using DSM-IV Axis I psychiatric disorders as gold standard. Psychiatry Research, 105, 243-253. doi:10.1016/S0165-1781(01)00329-8
[23] Silveira, E., Taft, C., Sundh, V., Waern, M., Palsson, S. and Steen, B. (2005) Performance of the SF-36 health survey in screening for depressive and anxiety disorders in an elderly female Swedish population. Quality of Life Research, 14, 1263-1274. doi:10.1007/s11136-004-7753-5

[24] Lesman-Leegte, I., Jaarsma, T., Sanderman, R., Linssen, G. and van Veldhuisen, D.J. (2006) Depressive symptoms are prominent among elderly hospitalised heart failure patients. European Journal of Heart Failure, 8, 634-640. doi:10.1016/j.ejheart.2005.11.010

[25] Redeker, N.S. (2006) Somatic symptoms explain differences in psychological distress in heart failure patients vs a comparison group. Progress in Cardiovascular Nursing, 21, 182-189. doi:10.1111/j.0889-7204.2006.05643.x

[26] Vitiello, M.V., Rybarczyk, B., Von Korff, M. and Stepanski, E.J. (2009) Cognitive behavioral therapy for insomnia improves sleep and decreases pain in older adults with co-morbid insomnia and osteoarthritis. Journal of Clinical Sleep Medicine, 5, 355-362.

[27] Cully, J.A., Stanley, M.A., Deswal, A., Hanania, N.A., Phillips, L.L. and Kunik, M.E. (2010) Cognitive-behavioral therapy for chronic cardiopulmonary conditions: Preliminary outcomes from an open trial. Primary Care Companion to the Journal of Clinical Psychiatry, 12, PCC.09m00896.

[28] Sullivan, M.J., Wood, L., Terry, J., et al. (2009) The support, education, and research in chronic heart failure study (SEARCH): A mindfulness-based psychoeducational intervention improves depression and clinical symptoms in patients with chronic heart failure. American Heart Journal, 157, 84-90. doi:10.1016/j.ahj.2008.08.033

[29] Lundh, L.G. and Broman, J.E. (2000) Insomnia as an interaction between sleep-interfering and sleep-interpreting processes. Journal of Psychosomatic Research, 49, 299310. doi:10.1016/S0022-3999(00)00150-1

[30] Erickson, V.S., Westlake, C.A., Dracup, K.A., Woo, M.A. and Hage, A. (2003) Sleep disturbance symptoms in patients with heart failure. AACN Clinical Issues, 14, 477487. doi:10.1097/00044067-200311000-00009

[31] Pallesen, S., Nordhus, I.H., Kvale, G., et al. (2002) Psychological characteristics of elderly insomniacs. Scandinavian Journal of Psychology, 43, 425-432. doi:10.1111/1467-9450.00311

[32] Morin, C.M., Rodrigue, S. and Ivers, H. (2003) Role of stress, arousal, and coping skills in primary insomnia. Psychosomatic Medicine, 65, 259-267. doi:10.1097/01.PSY.0000030391.09558.A3

[33] Johansson, P., Alehagen, U., Svensson, E., Svanborg, E., Dahlstrom, U. and Brostrom, A. (2010) Determinants of global perceived health in community-dwelling elderly screened for heart failure and sleep-disordered breathing. Journal of Cardiovascular Nursing, 25, E16-E26.

[34] Devine, E.B., Hakim, Z. and Green, J. (2005) A systematic review of patient-reported outcome instruments measuring sleep dysfunction in adults. Pharmacoeconomics, 23, 889-912. doi:10.2165/00019053-200523090-00003

[35] Netzer, N.C., Stoohs, R.A., Netzer, C.M., Clark, K. and Strohl, K.P. (1999) Using the Berlin questionnaire to 
identify patients at risk for the sleep apna syndrome. Annals of Internal Medicine, 131, 485-491.

[36] Petit, L., Azad, N., Byszewski, A., Sarazan, F.F. and Power, B. (2003) Non-pharmacological management of primary and secondary insomnia among older people: Review of assessment tools and treatments. Age Ageing, 32, 19-25. doi:10.1093/ageing/32.1.19

[37] Jaussent, I., Bouyer, J., Ancelin, M.L., et al. (2011) Insomnia and daytime sleepiness are risk factors for depressive symptoms in the elderly. Sleep, 34, 1103-1110.

[38] Espie, C.A. (2009) "Stepped care": A health technology solution for delivering cognitive behavioral therapy as a first line insomnia treatment. Sleep, 32, 1549-1558.

[39] Morin, C.M., Bootzin, R.R., Buysse, D.J., Edinger, J.D., Espie, C.A. and Lichstein, K.L. (2006) Psychological and behavioral treatment of insomnia: Update of the recent evidence (1998-2004). Sleep, 29, 1398-1414.

[40] Strom, L., Pettersson, R. and Andersson, G. (2004) Internet-based treatment for insomnia: A controlled evaluation. Journal of Consulting and Clinical Psychology, 72, 113-

\section{0. doi:10.1037/0022-006X.72.1.113}

[41] Andersson, G. (2009) Using the Internet to provide cognitive behaviour therapy. Behaviour Research and Therapy, 47, 175-180. doi:10.1016/j.brat.2009.01.010

[42] Andrews, G., Cuijpers, P., Craske, M.G., McEvoy, P. and Titov, N. (2010) Computer therapy for the anxiety and depressive disorders is effective, acceptable and practical health care: A meta-analysis. PLoS One, 5, e13196. doi:10.1371/journal.pone.0013196

[43] Williams, Jr., J.W., Pignone, M., Ramirez, G. and Perez Stellato, C. (2002) Identifying depression in primary care: A literature synthesis of case-finding instruments. General Hospital Psychiatry, 24, 225-237. doi:10.1016/S0163-8343(02)00195-0

[44] Ulander, M., Arestedt, K., Svanborg, E., Johansson, P. and Broström, A. (2013) The fairness of the Epworth sleepiness scale: Two approaches to differential item functioning. Sleep Breath, 17, 157-165. doi:10.1007/s11325-012-0664-8 